\title{
Prolonged millimeter-wave radio emission from a solar flare near the limb
}

\author{
S. Pohjolainen ${ }^{1,2}$, J. Hildebrandt ${ }^{3}$, M. Karlický ${ }^{4}$, A. Magun ${ }^{5}$, and I. M. Chertok ${ }^{6}$ \\ 1 Tuorla Observatory, Väisälä Institute for Space Physics and Astronomy, 21500 Piikkiö, Finland \\ 2 Observatoire de Paris, DASOP, 92195 Meudon, France \\ 3 Astrophysical Institute Potsdam, 14482 Potsdam, Germany \\ 4 Astronomical Institute Ondřejov, 25165 Ondrejov, Czech Republic \\ 5 Institute of Applied Physics, 3012 Bern, Switzerland \\ ${ }^{6}$ IZMIRAN, Russian Academy of Sciences, Troitsk, Moscow Region, 142190 Russia
}

Received 3 November 2000 / Accepted 26 September 2002

\begin{abstract}
We present a multi-wavelength analysis of a gradual radio flare on June 27, 1993 which showed emission at millimeter waves long after the soft X-ray flux had peaked. The radio flare located at S12 E75 was associated with a GOES class M3.6 flare that lasted for more than one hour and hard X-ray emission during the rising phase of the soft X-ray/radio emission. The maximum radio flux density at $35 \mathrm{GHz}$ was $60 \mathrm{sfu}$, but the calculated thermal bremsstrahlung flux from the GOES soft $\mathrm{X}$-rays was less than half of that. The possible explanations for this prolonged millimeter wave emission could be accelerated high-energy electrons gyrating along the field-lines (nonthermal gyrosynchrotron emission) or thermal bremsstrahlung from evaporating chromospheric warm and dense plasma (cool enough to go undetected by GOES), or a mixture of these. Our model calculations show that even an inhomogeneous source containing both kinds of particles would not be able to produce such a spectral shape. A second source with extremely high electron densities $\left(>10^{16} \mathrm{~m}^{-3}\right)$, large source dimensions $\left(>10^{15} \mathrm{~m}^{2}\right)$, and very low temperatures $\left(<10^{6} \mathrm{~K}\right)$ must be assumed to explain the observed radio spectra.
\end{abstract}

Key words. Sun: chromosphere - Sun: corona - Sun: flares - Sun: radio radiation - X-rays: general

\section{Introduction}

In many radio flares observed at millimeter and centimeter wavelengths a gradual thermal component starts to dominate after the impulsive phase, in agreement with the evolution in soft X-rays (Kundu et al. 1994; Pohjolainen et al. 1996). Also, radio flares that peak long after the soft $\mathrm{X}$-ray flux maximum have been observed (Kundu et al. 1994; Pohjolainen et al. 1997). The statistical study by Pohjolainen et al. (1996) that analysed all the Metsähovi solar radio bursts from 1989-1992 showed that in 15 out of the analysed 34 gradual-type bursts (i.e., radio emission that shows a gradual rise and fall burst with a duration longer than $10 \mathrm{~min}$ ) the flux curves could be explained solely by thermal bremsstrahlung. Also, in 23 of the events the time of the calculated thermal bremsstrahlung maximum coincided with the observed burst maximum, even if the flux densities did not completely agree.

A comparison between five Metsähovi 37-GHz gradual radio events and the corresponding CGRO BATSE hard X-ray events (Pohjolainen et al. 1997) showed again how two events agreed perfectly with a thermal bremsstrahlung explanation but three did not (neither in flux density nor in temporal evolution).

Send offprint requests to: S. Pohjolainen, e-mail: spo@astro.utu.fi
These three were also associated with hard X-ray emission that was very impulsive in the beginning of the event but then decayed gradually. One of these events (February 15, 1992) was a small ( $3 \mathrm{sfu}$ ) rise-and-fall event (duration $<10 \mathrm{~min}$ ), and therefore does not fully fit into the category of gradual radio flares that normally have much larger fluxes and longer duration, but the other two are listed in Table 1 (July 7, 1992, and June 27, 1993). To see how common millimeter-wave rich gradual flares really are, the Nobeyama Radioheliograph Web Archive (17 and $34 \mathrm{GHz}$ observations) was searched. From their 1993-2000 solar radio data only one gradual event was found that showed more intense emission at millimeter waves than at centimeter waves (July 9, 2000). This event happened during Yohkoh night time (as did the five Metsähovi events described earlier) and also by this time CGRO had stopped observing. The main characteristics of the three millimeter-wave rich events (two Metsähovi, one Nobeyama) are summarised in Table 1.

We present here a multi-wavelength analysis of the June 27 , 1993 event. It was selected on the basis that it has the largest number of data available of the three. The beginning of the flare was imaged by Yohkoh, although the main gradual radio phase happened during Yohkoh night time. We discuss the interpretation of the radio spectrum, and compare the millimeter wavelength observations with other wavelength data. We also 
Table 1. Millimeter-wave rich gradual solar radio flares.

\begin{tabular}{rrrrr}
\hline \hline Date & Start time & Radio flare location & 37/34 GHz flux & GOES class \\
\hline July 7, 1992 & $11: 32 \mathrm{UT}$ & S10 E80 & $35 \mathrm{sfu}$ & M4.1 \\
June 27, 1993 & $11: 14 \mathrm{UT}$ & S12 E75 & $38 \mathrm{sfu}$ & M3.6 \\
July 9, 2000 & 04:15 UT & N18 E72 & $115 \mathrm{sfu}$ & C3(?) \\
\hline
\end{tabular}

suggest possible explanations for the long-lasting, high millimeter-wave flux, although in the absence of simultaneous imaging and hard X-ray observations for the complete duration of the flare, this remains somewhat speculative.

\section{Observations}

\subsection{Instruments}

The June 27, 1993 flare that occurred between 11:00 and 12:00 UT was observed with several radio instruments at millimeter and centimeter (i.e. microwave) wavelengths (Bern and Metsähovi) and at decimeter/meter wavelengths (Ondrejov, IZMIRAN, and Nancay), see Fig. 1. We used the full disk flux density observations from Bern at 8.4, 11.8, 19.6, and $35.0 \mathrm{GHz}$, with $0.1 \mathrm{~s}$ time resolution. Active region tracking with $0.05 \mathrm{~s}$ time resolution was obtained from Metsähovi at $37 \mathrm{GHz}$. The 2.4 arcmin beam of the telescope was pointed to the active region (S12 E75) and the flux density from that area was recorded. Full disk flux density observations at $3 \mathrm{GHz}$ from Ondrejov (0.01 s time resolution) and from IZMIRAN (0.04 s time resolution) were used to construct radio spectra during the flare.

IZMIRAN dynamic spectra at $45-180 \mathrm{MHz}$ (recorded on film in 1993) were used for detecting spectral drifts during or after the flare. Nancay radioheliograph East-West and NorthSouth solar disk scans at five selected frequencies in the 164$435 \mathrm{MHz}$ frequency range were analysed to determine the burst source locations. The Nancay radioheliograph has a $0.5 \mathrm{~s}$ time resolution at each frequency. The radio fluxes reported in the Solar Geophysical Data Reports were checked for comparison. CGRO BATSE observed enhanced hard X-ray count rates in the two lowest energy channels of the instrument (DISCLA rate every $1.024 \mathrm{~s}$ ), between 25 and $100 \mathrm{keV}$. BATSE solar viewing times are indicated in Fig. 1. GOES satellites provided the soft X-ray fluxes in $3 \mathrm{~s}$ time resolution, and Yohkoh SXT the soft X-ray images (image cadence depends on the observing mode). Yohkoh HXT hard X-ray counts ( $0.5 \mathrm{~s}$ time resolution) were observed mainly in the $14-53 \mathrm{keV}$ energy range. Yohkoh observations were interrupted very early in the event due to satellite night time, see Fig. 2.

\subsection{Microwave/millimeter wave observations}

Figure 2 shows the Metsähovi single frequency observations at $37 \mathrm{GHz}(8 \mathrm{~mm})$ during 11:15-12:15 UT. The flux density was recorded from a beam area with a diameter of 2.4 arcmin which was pointed to the active region. The figure shows a gradual rise and fall event with a flux maximum around 11:33 UT. The calculated amount of thermal radio bremsstrahlung
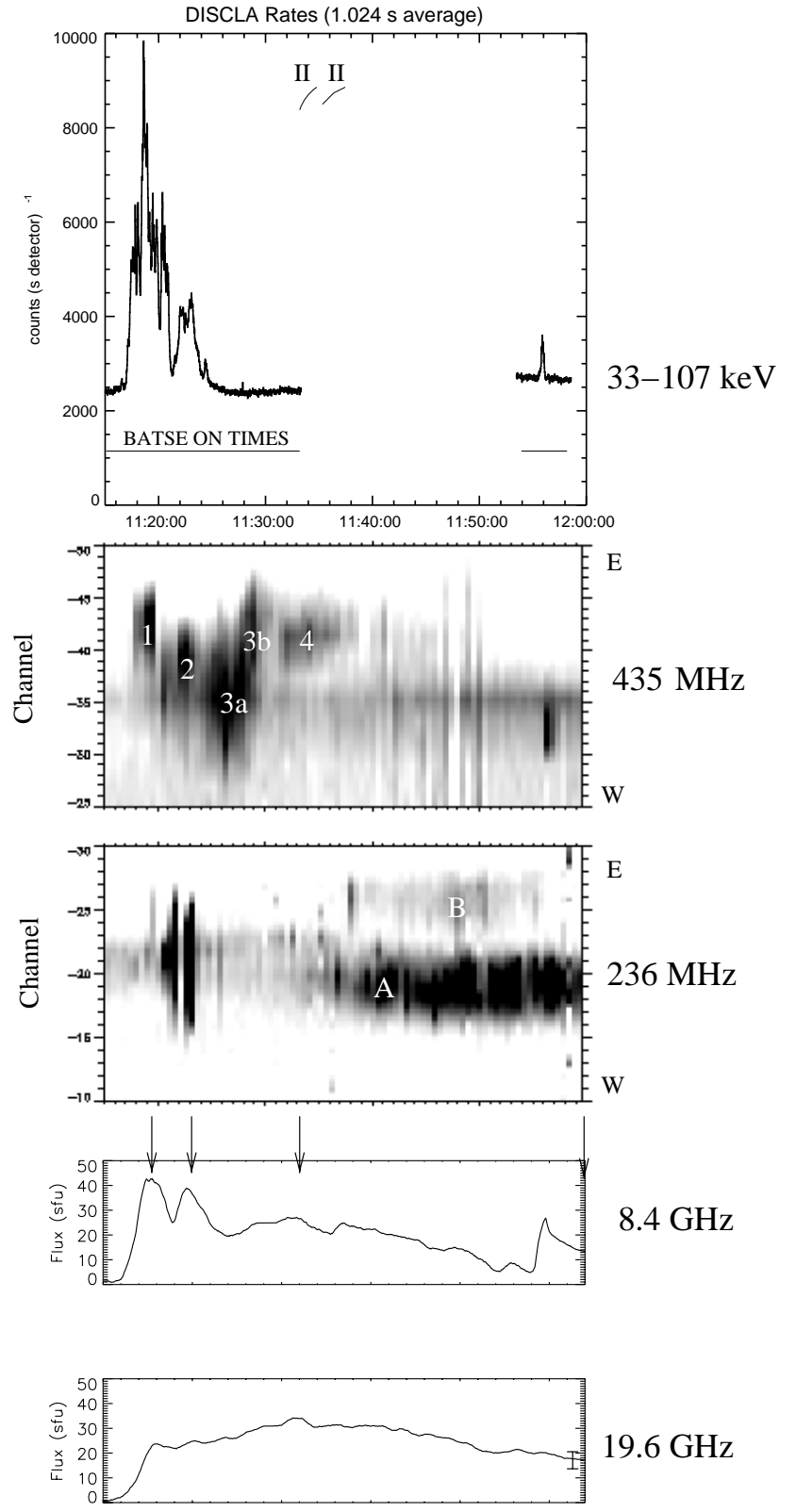

Fig. 1. Hard X-ray and radio data from June 27, 1993 at 11:1512:00 UT. BATSE count rates in the 33-107 keV energy range (top), Nancay Radioheliograph East-West scans over the solar disk at 435 and $236 \mathrm{MHz}$ (middle), and Bern full disk flux at 8.4 and $19.6 \mathrm{GHz}$ (bottom). The times of the strongest type II emissions are depicted in the plot at the top. The 2-D source positions of the 1-D temporal features 1, 2, 3, and 4 (at $435 \mathrm{MHz}$ ), and of $\mathrm{A}$ and $\mathrm{B}$ (at $236 \mathrm{MHz}$ ) are given in Fig. 3. Arrows mark the times of the spectral plots in Fig. 7. The error bar in the $19.6 \mathrm{GHz}$ plot marks the maximum error in flux density at that frequency around 12 UT. The error is less at earlier times and at lower frequencies. 


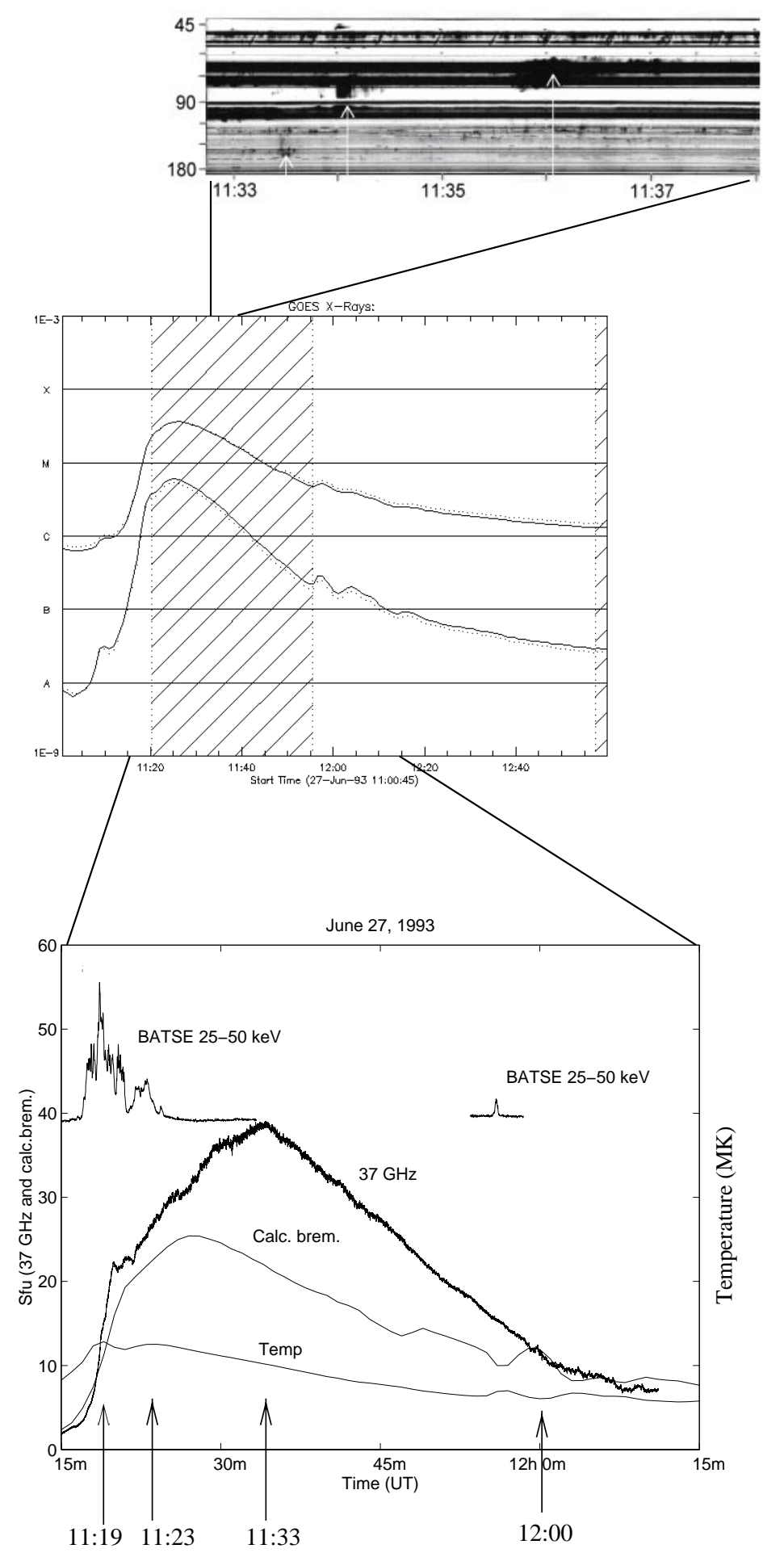

Fig. 2. From top to bottom: IZMIRAN dynamic spectra at 45-180 MHz at 11:33-11:38 UT on June 27, 1993. Arrows mark the strongest fragmented type II radio emission features in the plot. The times of the strongest type II features are also marked in Fig. 1. GOES soft X-rays on June 27, 1993 during 11:00-13:00 UT, where the shaded areas represents Yohkoh satellite night time. Metsähovi 37 GHz flux from the active region area (2.4 arcmin beam area) during 11:15-12:15 UT. The "calc. brem." curve shows the calculated amount of thermal bremsstrahlung at the same frequency, and "temp" the calculated plasma temperature (from GOES soft X-ray flux, assuming isothermal plasma density). Arrows mark the times of the spectral plots in Fig. 7.

("calc. brem." in Fig. 2) at $37 \mathrm{GHz}$ is seen to be significantly less than what was observed. The calculation (see Thomas et al. 1985; Pohjolainen et al. 1996) assumes an isothermal plasma density model which does not necessarily reflect the true conditions on the Sun, but gives an approximation. The GOES observations are reliable within $5 \%$ for plasma temperatures in the range of 5-30 MK, but the temperature is known to be a monotonic function of the flux ratio down to a $1 \mathrm{MK}$ 
temperature - i.e., we can still determine if the temperature and emissivity of the plasma are increasing or decreasing (Bornmann 1990).

The bottom panel in Fig. 1 presents the Bern full disk flux density observations at 8.4 and $19.6 \mathrm{GHz}$, corrected for atmospheric transmission and integrated over $60 \mathrm{~s}$. At microwaves (8.4 GHz), two distinct flux maxima are visible around 11:19 and 11:23 UT (times marked by the first two arrows in Fig. 1). The frequency in between microwaves and millimeter waves (19.6 GHz) shows the first maxima, but then the flux rises gradually and does not peak until 11:33 UT (third arrow in Fig. 1).

The $19 \mathrm{GHz}$ flux maximum occurred some $8 \mathrm{~min}$ after the GOES soft X-ray flux maximum (at 11:25 UT), and a few minutes after the $\mathrm{H} \alpha$ flare maximum (at 11:29 UT). The radio emission at $3 \mathrm{GHz}$ (Ondrejov and Izmiran) showed good correlation with the hard X-rays up to the time when BATSE entered night time at 11:33 UT, and by 13:50 UT the radio flux had fallen to the pre-flare level.

The observed radio spectra at 11:19 UT (first hard X-ray and microwave burst), 11:23 UT (second hard X-ray and microwave burst), 11:33 UT (19/35/37 GHz flux maximum), and 12:00 UT (decline phase of the flare) are plotted in Fig. 7 (symbols). The first spectrum at 11:19 UT shows a typical radio burst spectrum with a turn-over frequency around $5 \mathrm{GHz}$. The second spectrum at 11:23 UT shows how the radio flux at higher frequencies has started to rise. After 11:23 UT the $35 \mathrm{GHz}$ radio flux exceeds the lower frequency fluxes, and the radio spectrum at 11:33 UT has a rising slope towards the high frequencies. Due to the small number of data points it is difficult to determine the spectral peak frequency, but it could be higher than $37 \mathrm{GHz}$. In the declining phase at 12:00 UT the radio spectrum is still dominated by high-frequency radiation.

The Bern $35 \mathrm{GHz}$ flux was somewhat higher than the Metsähovi $37 \mathrm{GHz}$ flux (Bern flux maximum 60 sfu, Metsähovi $40 \mathrm{sfu}$ ). Both Bern and Metsähovi fluxes are calculated relative to the quiet Sun level, but the Bern high frequency observations are sometimes affected by slow drifts in the atmospheric absorption. This drift is attributed to a slow decrease in the atmospheric transmission. The 8.4 and $19.6 \mathrm{GHz}$ burst flux profiles presented in Fig. 1 were corrected for this effect. However, at $35 \mathrm{GHz}$ where the drift in flux is much larger it becomes very difficult to do reliable flux corrections after 11:33 UT. For the $19.6 \mathrm{GHz}$ flux an error of less than $\pm 25 \%$ at $12 \mathrm{UT}$ can be given. For $35 \mathrm{GHz}$ the lower limit of the peak flux is $60 \mathrm{sfu}$. For the Metsähovi $37 \mathrm{GHz}$ observations the flux error due to instrumental effects is less than $0.1 \mathrm{sfu}$. The recording was done on a clear, dry, and sunny day near noon time (highest elevation), which gives the best possible observing conditions. Full disk solar maps were recorded before and after the 2 hour tracking period for calibration purposes, and they did not show any significant variation in atmospheric transmission. The optical depth is different at different antenna (target) elevations (see, e.g., Pohjolainen \& Urpo 1997 for Metsähovi observations) and in this case the flux error due to the atmosphere should be less than $1 \mathrm{sfu}$. On the other hand, the Bern $35 \mathrm{GHz}$ and Metsähovi $37 \mathrm{GHz}$ flux curves also showed slightly different time evolution. An alternative explanation for the difference in the Metsähovi $37 \mathrm{GHz}$ and the Bern $35 \mathrm{GHz}$ flux is that
Metsähovi may have recorded only part of the flux, within the 2.4 arcmin beam centered over the active region, while Bern could have recorded the full flux (i.e., from a larger emitting area). If interpreted this way, the Metsähovi 40 sfu at $37 \mathrm{GHz}$ would give the lower flux limit.

\subsection{Decimeter/meter wave observations}

Decimetric emission at $435 \mathrm{MHz}$ shows bursts starting around 11:19, 11:23, 11:26, and 11:32 UT. The first two (labelled "1" and " 2 " in Fig. 1) coinside with the microwave flux density peaks at $8.4 \mathrm{GHz}$ and with the two hard X-ray count rate peaks. The third burst (labelled " $3 a$ " and " $3 b$ " in Fig. 1) does not seem to have any counterpart at other observed wavelengths, but the fourth (labelled "4" in Fig. 1) starts around the time of the millimeter wave flux maximum at 11:33 UT. The four above-mentioned bursts at $435 \mathrm{MHz}$ occured at different locations on the disk, seen in the Nancay radioheliograph images in Fig. 3: the first (source "1") was located near the limb but the next ones (sources " 2 " and " $3 a$ ") were closer to the soft X-ray flare site (labelled "F1" in Fig. 4) in the beginning of the flare. Sources " $3 b$ " and " 4 " were again located near the limb. From the soft X-ray images it looks as if the decimetric bursts followed some of the loop structures.

At metric wavelengths (164 and $236 \mathrm{MHz}$ ) two bursts were observed at separate locations on the disk, starting around 11:37 UT. Figure 1 shows the time and the 1-D spatial evolution of these bursts at $236 \mathrm{MHz}$ (sources "A" and "B" in the East-West scans). Figure 3 shows their source positions on the solar disk. The $236 \mathrm{MHz}$ flux source "A", in the rising phase around 11:37 UT, was very near the location where the soft X-ray flare started and most of the hard X-ray emission was coming from before Yohkoh night time set in (location labelled "F1" in Fig. 4). The fainter 'B' source was located over the limb (labelled "B" Fig. 3). Source "B" was visible until around 12 UT, and after that also the flux of source "A" started to decrease. At $164 \mathrm{MHz}$ a similar source to "B" was observed, at a larger distance from the limb.

At longer metric wavelengths, the dynamic spectra at 45$180 \mathrm{MHz}$ shows fragmented type II radio emission between 11:33 and 11:38 UT (IZMIRAN dynamic spectra in Fig. 2). Distinct frequency-drifting radio type II emission was observed around 11:34 UT just below $90 \mathrm{MHz}$ and around 11:36 UT at lower frequencies, indicating plasma emission at the local plasma frequency after a passing disturbance. These fragmented emission sources were too faint to be resolved in the Nancay $164 \mathrm{MHz}$ scans.

\subsection{X-ray observations}

The GOES soft X-rays show a class M3.6 flare during 11:1112:15 UT (Fig. 2). The GOES maximum was around 11:25 UT. In hard X-rays, a flare event was observed during 11:1611:33 UT by CGRO BATSE (observations interrupted by satellite night time at that point), see Fig. 1. Impulsive burst peaks superposed on the gradual component were observed from 11:17 UT until 11:25 UT, with two count rate peaks around 


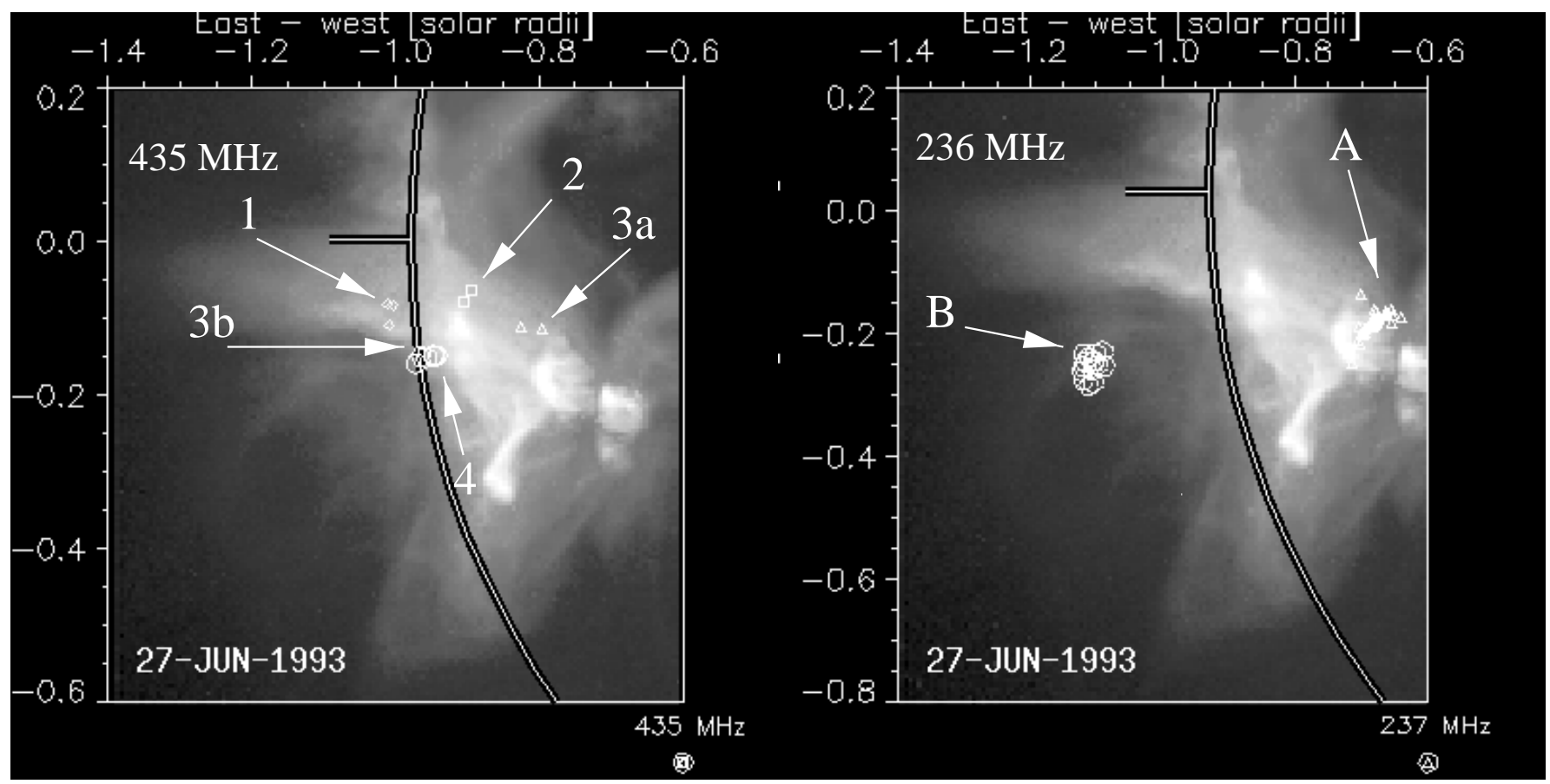

Fig. 3. Left: Yohkoh SXT soft X-ray image at 12:40 UT (post-flare), with radio source locations 1, 2, 3a, $3 \mathrm{~b}$, and 4 at $435 \mathrm{MHz}$. Right: radio source locations $\mathrm{A}$ and $\mathrm{B}$ at $236 \mathrm{MHz}$ over the Yohkoh image. The source locations correspond to the burst features seen in the spectra, presented in Fig. 1.

11:19 and 11:23 UT. After this more impulsive phase the hard $\mathrm{X}$-ray counts diminished gradually to the pre-flare level, except in the 33-56 keV energy range (BATSE DISCLA) which continued to show some counts above the backgound level, with some hints of a rising count rate just before satellite night time set in. A short hard X-ray burst was observed near the time when the satellite started observing again, at 11:55 UT, in the late phase of the radio flare. The Yohkoh hard X-ray images, Fig. 4, show an extended source with a diameter of about 1 arcmin near the time of the first flux peak at 11:19 UT.

\section{Possible explanations for the high mm-wave flux}

A super-hot thermal component can be ruled out because of the spectral distribution in X-rays during the flare. Also the millimeter and microwave fluxes are too high for a super-hot component. This leaves basically two mechanisms that can create a high mm-wave flux without creating soft X-ray emission detectable by GOES: a) thermal bremmstrahlung from warm - or even cool ( $<1 \mathrm{MK})$ - and dense plasma and b) gyrosynchrotron emission by high-energy electrons. We will now look more closely into these two emission mechanisms.

a) Contribution of Coulomb bremsstrahlung from evaporating chromospheric warm $(T<5 \mathrm{MK})$ and dense plasma (undetected by GOES), filling up the microwave source and overcoming gyrosychrotron emission at high frequencies have been shown to be possible (Chertok et al. 1995; Urpo et al. 1986). The examples in Chertok et al. (1995) required large bursts with microwave fluxes larger than $100 \mathrm{sfu}$, and sufficiently intense soft X-ray emission correlated with the mm-wave bursts.
Separate flaring sites at different plasma temperatures cannot be ruled out as we do not have spatial information from the high mm-wave flux period. On the other hand, suppression and absorption effects would be needed to explain the low cmwave flux in comparison with the high mm-wave flux, as the radio bremsstrahlung spectrum should basically be flat. This would be difficult to achieve, as loops are less dense in the high corona. The difference in flux between the full disk and the 2.4 arcmin beam radio observations also suggest that the emission source was extended.

b) For gyrosynchrotron emission an increasing magnetic field strength and a decreasing electron power law index can move the spectral peak towards the higher frequencies, while changes in the source electron density and temperature alter the flux density, but do not significantly change the general spectral shape. Although gyrosynchrotron emissivity is a strong function of magnetic field strength $\left(\propto B^{3}\right)$, the emissivity also depends on the angle $\theta$ between the line of sight and the magnetic field $\left(\propto \sin ^{2} \theta\right)$, see e.g. Dulk (1985). An increase in $B$ can significantly lower the number of radio-emitting electrons required, but it also increases the spectral peak frequency - which is consistent with our observations. Loop footpoints would therefore be bright at millimeter waves (stronger magnetic fields), and a favourable viewing angle near the limb would further enhance the emissivity.

In impulsive solar flares, the cm-wave emission is claimed to be produced by electrons in the range $100 \mathrm{keV}-1 \mathrm{MeV}$ and the mm-wave emission by electrons with energies of $0.5 \mathrm{MeV}$ and above (Kundu et al. 1994). In the case where we have prolonged but weak acceleration of electrons up to $1 \mathrm{MeV}$ - these electrons will be trapped in flare loops for the time proportional 

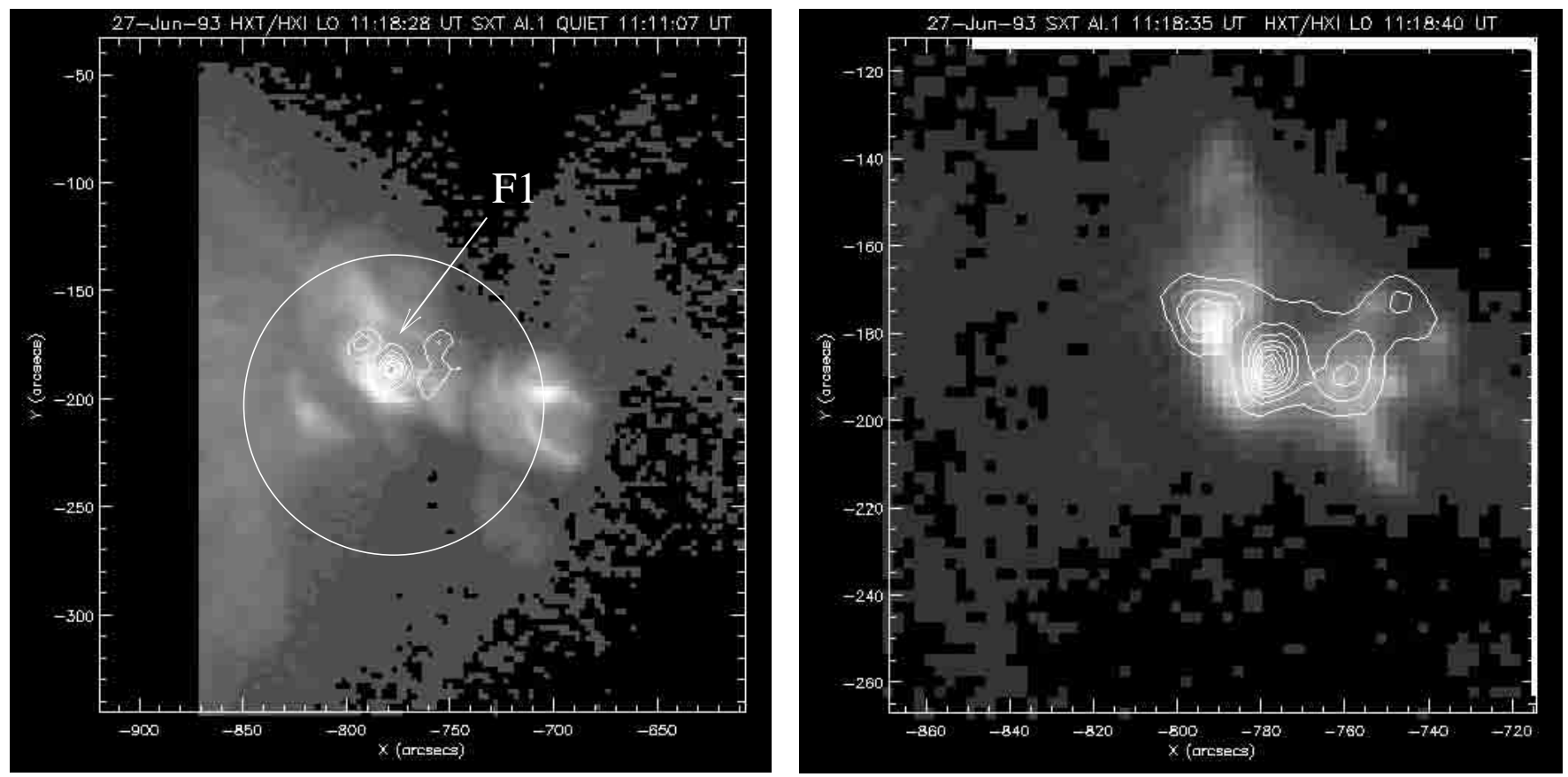

Fig. 4. Left: Yohkoh HXT 14-23 keV hard X-ray sources at 11:18:28 UT (contours) over the Yohkoh SXT soft X-ray image at 11:11:07 UT. "F1" points to the location where the soft X-ray flare started and most of the hard X-ray emission was coming from before Yohkoh night time set in. The white circle represents the Metsähovi 2.4 arcmin radio beam, pointed to the active region and recording the total flux density from that area. Right: Yohkoh HXT 14-23 keV hard X-ray sources at 11:18:40 UT (contours) over the Yohkoh SXT soft X-ray image at 11:18:35 UT. The hard X-ray sources observed in the 14-53 keV energy range were located in the sigmoid-shaped flaring region. In later images the hard $\mathrm{X}$-ray source was elongated into a loop-like structure with a diameter around $1 \mathrm{arcmin}$.

to $E^{3 / 2} N_{\mathrm{e}}^{-1}$ (Aschwanden et al. 1997) - the energy of the electrons is collisionally decreasing as $\frac{\mathrm{d} E}{\mathrm{~d} t}=-\frac{2 \pi e^{4} \Lambda}{E} N_{\mathrm{e}} v$, where $E$ is the energy of the electron, $e$ is the electron charge, $\Lambda$ is the Coulomb logarithm, $N_{\mathrm{e}}$ the electron density of the surrounding plasma, and $v$ is the electron speed (Emslie 1978). From these formulas we see that $\mathrm{keV}$ electrons can be accumulated for a long time only in a low-density corona where their collisional losses are low. Trapping times for keV electrons are in the range of several tens of seconds. For mm-wave emitting electrons the corresponding upper limit for trapping times can be several tens of minutes (Aschwanden et al. 1997). Prolonged acceleration will be needed to explain both the decimetric-metric emission as well as the high mm-wave flux. However, the small amount of hard X-rays after the impulsive phase and during the millimeter flux rise poses a problem. It has been shown previously (Raulin et al. 1999) that a small number of MeV electrons trapped in a loop can produce mm-wave emission with a hard $\mathrm{X}$-ray flux not likely to be detected by BATSE. The precipitation flux of the $\mathrm{keV}$ and $\mathrm{MeV}$ electrons would be low during trapping - as long as electrons are trapped they can produce $\mathrm{cm}$ - and mm-wave emission, but if they radiate hard X-rays they are lost from the system (Hudson \& Ryan 1995).

\section{Results of model calculations}

\subsection{Model description}

The model used for the calculations in order to fit the observed spectra is geometrically rather simple and will be only briefly described here. A more detailed description can be found in
Hildebrandt et al. (1998). It calculates the escaping intensity for each wanted frequency solving the equation of radiative transfer along the line of sight. In the case of a homogeneous source this is similar to the procedures of, e.g., Benka \& Holman (1992) or Ramaty (1969). For inhomogeneous sources we use a multi-layer model, i.e., the distributions for all parameters necessary to calculate the coefficients must be given along the ray trajectory. As relevant emission processes we consider gyrosynchrotron radiation (GSR) from nonthermal power-law (PL) electrons and gyroresonance absorption (GRA) as well as Coulomb-bremsstrahlung (CBS) from the thermal background plasma. Special attention was focussed on the computation of the GSR emission and absorption coefficients (full code, no approximation formulae). Polarization effects are also included but not considered here. Assuming a "single" PL distribution function for the nonthermal particles, the following parameters are finally needed for the calculation:
$N_{\mathrm{e}} \quad-\quad$ number density of the thermal (background) plasma
$B \quad-\quad$ magnetic field strength
$\theta \quad-\quad$ angle between the magnetic field vector and the line of sight
$L \quad-\quad$ thickness of the emission volume (in the line-of-sight direction)
$T \quad-\quad$ temperature of the (background) plasma
$N \quad-\quad$ number density of the nonthermal electrons
$E_{2} \quad-\quad$ high-energy boundary of the PL distribution
$\lambda-$ PLindex. 
Table 2. Maximum (or minimum) parameter values inside the hatched region in Figs. 5 and 6 used to model the four radio spectra in Fig. 7. Further explanations are given in the text.

\begin{tabular}{|c|c|c|c|c|c|c|c|c|c|}
\hline & & \multicolumn{2}{|c|}{ 11:19 UT } & \multicolumn{2}{|c|}{ 11:23 UT } & \multicolumn{2}{|c|}{ 11:33 UT } & \multicolumn{2}{|c|}{ 12:00 UT } \\
\hline & & CNTS & EXTS & CNTS & EXTS & CNTS & EXTS & CNTS & EXTS \\
\hline$B$ & {$[\mathrm{G}]$} & $315 \ldots 177$ & $141 \ldots 40$ & $315 \ldots 177$ & $141 \ldots 40$ & $315 \ldots 177$ & $141 \ldots 40$ & $315 \ldots 177$ & $141 \ldots 40$ \\
\hline$N_{\mathrm{e}}$ & {$\left[10^{15} \mathrm{~m}^{-3}\right]$} & $<12$ & $<20$ & $<12$ & $<32$ & $<12$ & $<40$ & $<8.9$ & $<33$ \\
\hline$\theta$ & {$\left[{ }^{\circ}\right]$} & $42 \ldots 47$ & $50 \ldots 40$ & $42 \ldots 47$ & $50 \ldots 40$ & $42 \ldots .47$ & $50 \ldots 40$ & $42 \ldots 47$ & $50 \ldots 40$ \\
\hline$T_{\mathrm{e}}$ & {$\left[10^{6} \mathrm{~K}\right]$} & $<13$ & $>0.6$ & $<13$ & $>0.59$ & $<13$ & $>0.54$ & $<13$ & $>0.54$ \\
\hline$L$ & {$\left[10^{6} \mathrm{~m}\right]$} & 8 & 20 & 8 & 20 & 8 & 20 & 8 & 20 \\
\hline$A_{\text {eff }}$ & {$\left[10^{14} \mathrm{~m}^{2}\right]$} & 1.6 & 7 & 1.6 & 9 & 1.6 & 13.5 & 1.2 & 6 \\
\hline$N$ & {$\left[10^{10} \mathrm{~m}^{-3}\right]$} & $<36$ & - & $<24$ & - & $<7.1$ & - & $<4.4$ & - \\
\hline$\lambda$ & & $6.1 \ldots 2.4$ & - & $6.1 \ldots 2.4$ & - & $6.1 \ldots 2.4$ & - & $6.1 \ldots 2.4$ & - \\
\hline$E_{1}$ & {$[\mathrm{keV}]$} & $3 . . .20$ & - & 3...20 & - & 3...20 & - & 3...20 & - \\
\hline$E_{2}$ & {$[\mathrm{MeV}]$} & $1 \ldots 15$ & - & $1 \ldots 15$ & - & $1 \ldots 15$ & - & $1 \ldots 15$ & - \\
\hline
\end{tabular}

(The low-energy boundary $E_{1}$ is already determined by the temperature and the ratio of number densities $N / N_{\mathrm{e}}$ using the normalization condition for the distribution functions; $\mathrm{cf}$. Hildebrandt et al. 1998 for more details.)

To compare the calculated intensity values with real observed spectra we have to convert them into flux density units by assuming an effective emission area $A_{\text {eff. }}$. So we have finally 9 free parameters which gives us enough space to play with them. On the other hand, every additional information is highly welcome and must be used in order to restrict the number of free parameters. It should be also mentioned here that a constant $A_{\text {eff }}$ is mearly a rough assumption which gives us only an upper limit for the resulting flux. But a (more realistic) numerical integration over the relevant area assuming threedimensional inhomogeneity would take too much time and efforts, in particular, if many test calculations are necessary.

\subsection{Results}

All attemps to fit the four considered spectra assuming a single homogeneous source failed except for the first one. Only at 11:19 UT the spectrum has a more or less "typical" gyrosynchroton shape, i.e. it decreases towards higher frequencies after its spectral maximum at about $2-5 \mathrm{GHz}$ (see Fig. 7 top left). For example, assuming $B=200 \mathrm{G}, N_{\mathrm{e}}=5 \times 10^{15} \mathrm{~m}^{-3}, T=10^{7} \mathrm{~K}$, $\theta=45^{\circ}, L=8 \times 10^{6} \mathrm{~m}, N=2 \times 10^{11} \mathrm{~m}^{-3}\left(N / N_{\mathrm{e}}=4 \times 10^{-5}\right)$, $\lambda=2.4$, and $E_{2}=15 \mathrm{MeV}$, one obtains - at least for $v>3 \mathrm{GHz}$ - a spectrum which would satisfactorily fit the observed one. However, the assumed values for the energetic particles $(\lambda$ and $\left.E_{2}\right)$ are already rather extreme and not necessarily typical for solar flare conditions. Considering the next spectra, it becomes clear that their shapes cannot be explained assuming homogenous and GSR dominated sources. The spectrum at 11:23 UT has a nearly constant flux in the range $10-30 \mathrm{GHz}$ and the observed spectrum at 11:30 UT shows even a clear increase up to $37 \mathrm{GHz}$ ! The (theoretically possible) case that the peak of the GSR spectrum is shifted towards such high frequencies due to strong $B$ inside the source must also turned down because this would require magnetic fields of about $5000 \mathrm{G}$ $\left(v_{\text {peak }} \propto B^{0.7 \ldots 0.8}\right)$ ! Increasing fluxes in this frequency range can only be caused by CBS from a very dense and not too hot $\left(<10^{6} \mathrm{~K}\right)$ plasma. However, the coexistence of such 'evaporated' plasma together with high-energy electrons in the framework of one inhomogeneous source (i.e. along one trajectory) brings a new serious problem: Either the low-frequency (GSR) part of the spectrum is totally suppressed by the thermal (CBS) part or the nonthermal source must be located so high in the corona that the magnetic field can hardly be strong enough (the thermal source must have a certain extension to produce fluxes of more than $30 \mathrm{sfu}$ ). Therefore, the only solution is to assume two neighboring sources (both located inside the antenna beam size of $2.4 \mathrm{arcmin}$ ) which are responsible together for the observed flux spectra. This does not contradict to spatially resolved observations at other wavelengths, for instance, to soft X-ray images (cf., e.g., Fig. 4).

The two sources finally used for the spectral fits are based on typical parameter profiles of the atmosphere above a sunspot, i.e., with $N_{\mathrm{e}} \sim 10^{15} \mathrm{~m}^{-3}$ and $T \sim 2 \times 10^{6} \mathrm{~K}$ at low coronal heights. The characteristic deviations from these values are defined to be concentrated on a certain region at about $25000 \mathrm{~km}$ height, where $N_{\mathrm{e}}$ is enhanced (for both sources). In the case of the "compact nonthermal source" (CNTS), this region has a vertical extension of $8000 \mathrm{~km}$ which corresponds to typical loop diameters. Also the magnetic field values are such as they will be found in loop structures near sunspots $(\sim 250 \mathrm{G}) . T$ is also enhanced there $\left(1.4 \times 10^{7} \mathrm{~K}\right.$ at the maximum) and nonthermal particles are defined to be present only inside this region. By contrast, the so-called "extended thermal source" (EXTS) is mainly characterized by larger extensions ( $20000 \mathrm{~km}$ in vertical direction) and a diminished temperature (down to $5.9 \times 10^{5} \mathrm{~K}$ ), which is, together with the enhanced electron density $\left(\$ 4 \times 10^{16} \mathrm{~m}^{-3}\right)$, necessary to produce the measured fluxes at $v>15 \mathrm{GHz}$. Such sources are possibly a result of chromospheric evaporation processes.

The basic characteristics (parameter profiles) are shown in Figs. 5 (CNTS) and 6 (EXTS). At the four considered times the curves are very similar and we show therefore only the most extreme case at 11:33 UT. The quantitative differences can be found in Table 2, where the extreme numbers (or ranges) are given for all four cases including the relevant effective areas. 


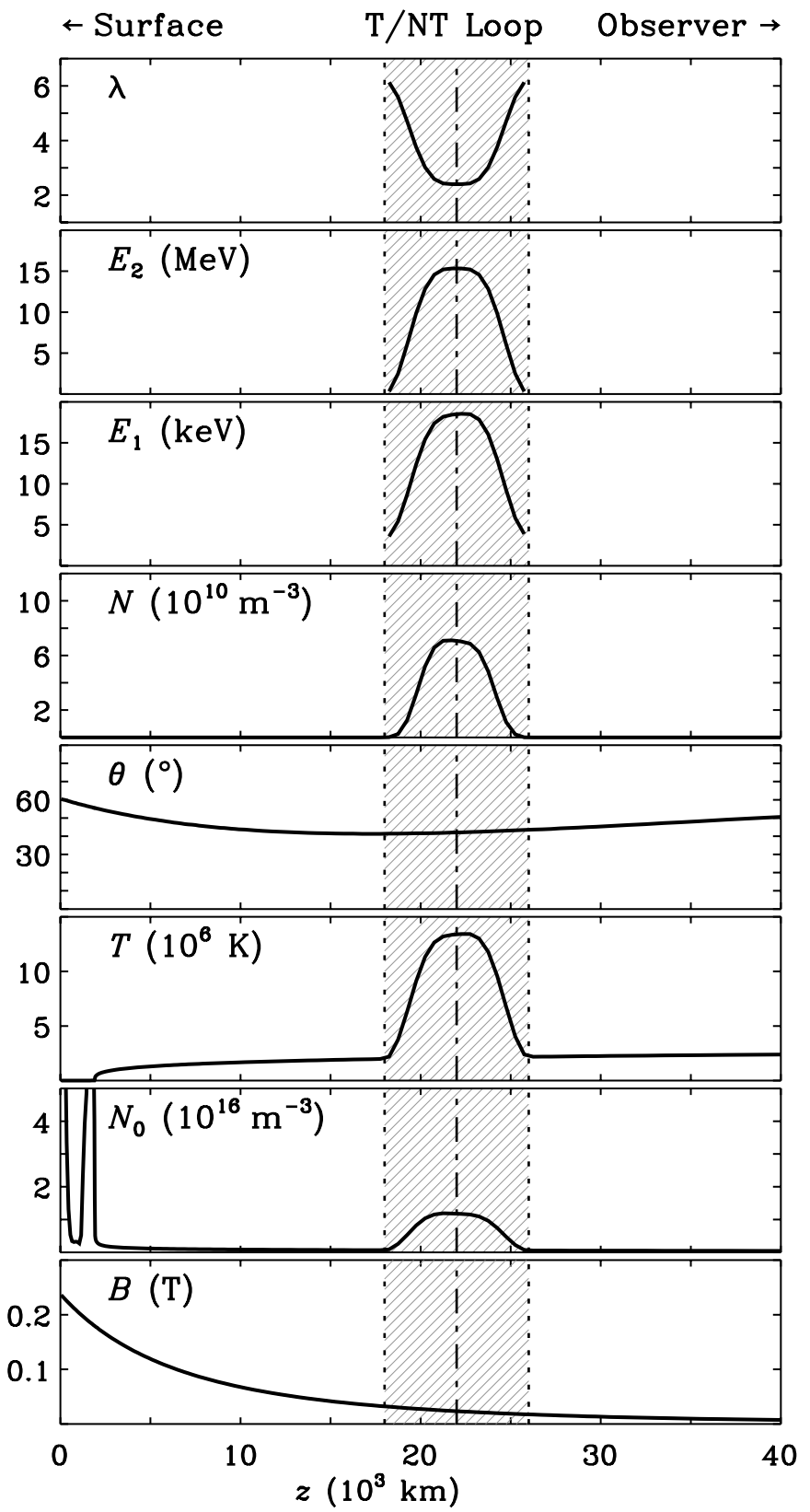

Fig. 5. Height profiles of the parameters in the compact nonthermal source (CNTS) used for the model calculations in order to interpret the observed spectrum at 11:33 UT. The hatched range marks the loop with thickness $L=8000 \mathrm{~km}$ located at $z=22000 \mathrm{~km}$ where temperature and density of the background plasma are enhanced. Only inside the loop nonthermal (power-law) electrons are assumed (top four panels). All parameters show a smooth behaviour, i.e., the most extreme (energetic) values occur around the center decreasing in direction to the loop boundary (no "jumps").

It should be mentioned that for our calculations nearly smoothed distributions (144 layers with variable thickness) were used, therefore, the "effective" (averaged) parameter values in the hatched regions may be more moderate than the given extrema suggest. Although our multi-layer model is rather time-consuming, the resultant spectra seem to justify such a procedure. They are smoother (especially in the low-frequency range) and many of the spectral features (harmonic structures)

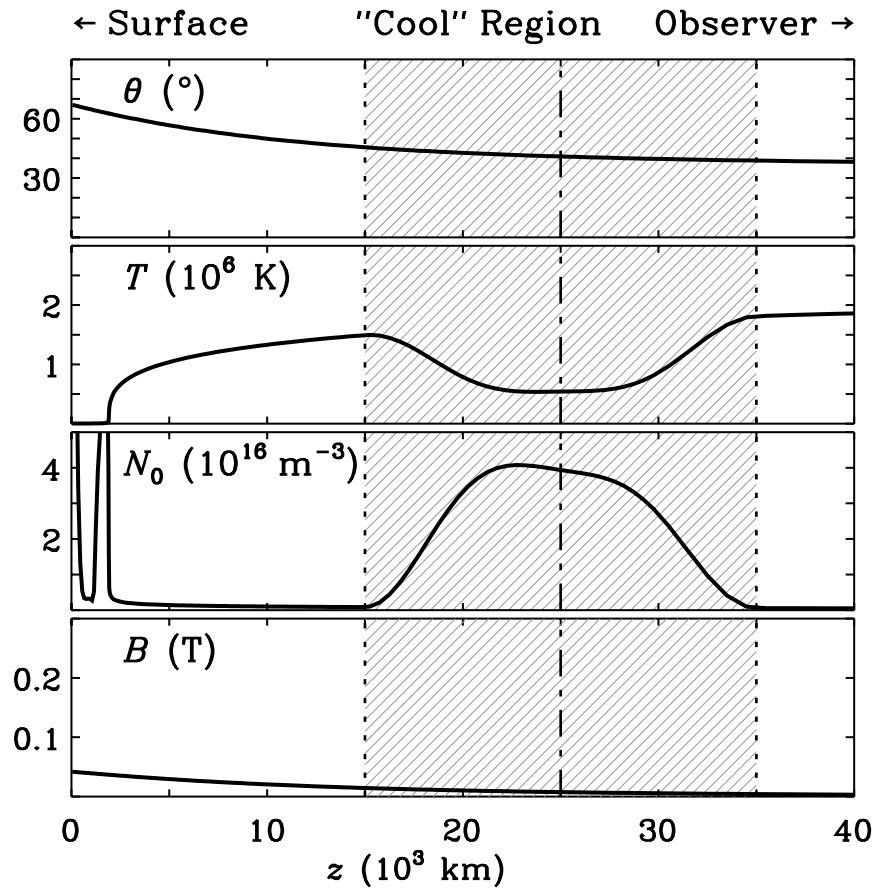

Fig. 6. Same as Fig. 5, but for the extended thermal source (EXTS) with (evaporated) cooler and more dense plasma inside the hatched region with thickness $L=20000 \mathrm{~km}$ centered at $z=25000 \mathrm{~km}$.

that occurred when simpler (and less realistic) models were used are suppressed now.

All calculated flux values in Fig. 7 are corrected to the quiet Sun, i.e., the flux coming from the quiet Sun was subtracted. The thin dashed and dashed-dotted curves represent the contributions from the two sources. It can clearly be seen that even at 11:33 and 12:00 UT the thermal source alone would not be able to produce the observed fluxes, i.e., at each time we need a second source. We do not claim that our parameter sets for CNTS are the only possible choices to fit the observed spectra. One can, of course, find parameter configurations for CNTS which are slightly different from ours and would also give a sufficient fit of the spectra. But we tried to avoid extreme numbers and use well-balanced values for our fits. Nevertheless, some parameters (in particular for EXTS) are rather difficult to explain, but their development shows at least a physically reasonable tendency: Whereas the number of PL electrons is slightly decaying after 11:19 UT, the density of the evaporated plasma reaches its maximum at 11:33 UT before it also starts to decrease.

\section{Discussion}

At the start of the analysed flare impulsive emission was observed in hard X-rays, cm-waves, and meter-decimeter waves. After this impulsive phase the hard X-ray counts dropped gradually, the flux at cm-waves started to decrease, and the mmwave flux continued to rise. The metric emission locations traced a large post-flare soft X-ray arcade for about $20 \mathrm{~min}$, indicating trapped electrons. During the metric emission the mmwave flux stayed high but started to decrease gradually. In comparison, the cm-wave flux had already gone down significantly 


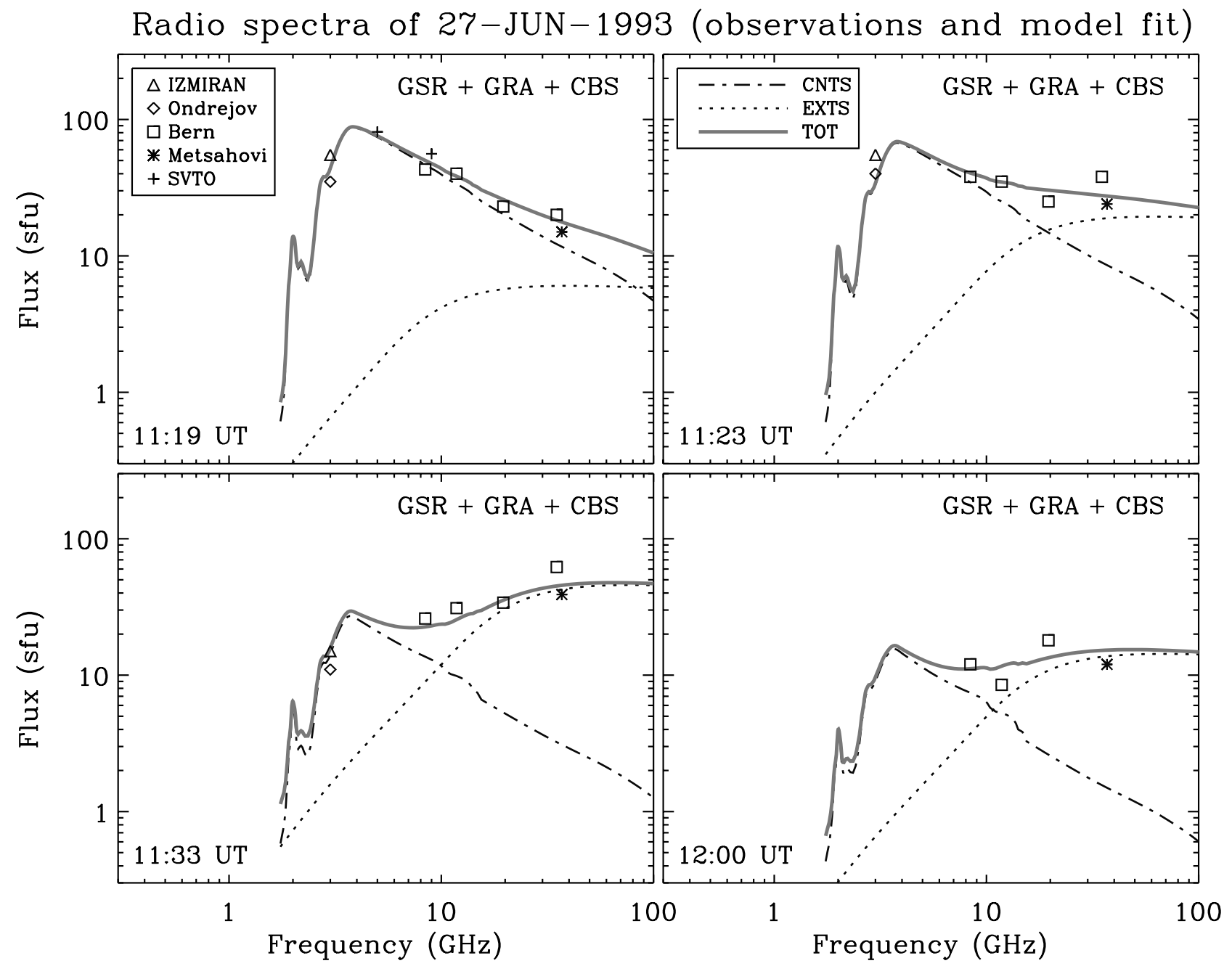

Fig. 7. Observed radio spectra at 11:19 UT, 11:23 UT, 11:33 UT, and 12:00 UT (symbols) and the relevant fits by our model calculations (lines). The solar fluxes are from different stations as given in the legend (top left). The Ondřejov, Bern, and Metsähovi radio fluxes are calculated relative to the quiet Sun level. The top-right legend explains the calculated fluxes: thin black lines show the contributions of the two different sources (CNTS - compact nonthermal source, EXTS - extended thermal source; see Table 2 and Figs. 5, 6). Thick gray lines refer to the resultant spectra. All flux values are corrected to the quiet Sun level. Further explanations are given in the text.

- in agreement with the calculated thermal bremsstrahlung flux from the cooling flare plasma.

The type II emission at $50-100 \mathrm{MHz}$, observed soon after the mm-wave flux peak, indicates a disturbance travelling in the solar atmosphere. Whether it was a shock wave, a plasmoid or a CME cannot be verified without simultaneous imaging observations. The observed decimetric bursts originated from widely separated locations, over complex loop systems near and over the limb, suggesting that some of the decimetric flaring activity might have been limb-occulted. The decimetric-metric emission can only originate in the high corona (at least 70000 $100000 \mathrm{~km}$ above the photosphere) where the magnetic field strengths and electron densities are low, and where the precipitating keV electrons can generate only negligible hard X-rays.

Radio emission at centimeter and millimeter waves is generally thought to originate at low heights, either from dense loops (thermal bremsstrahlung) or near the loop footpoints (gyrosynchrotron emission from places with strong magnetic fields). The difference between the observed mm-wave flux of the full-disk observations and the 2.4 arcmin collecting area observations may suggest that some of the mm-wave flux originated from an area larger than the 2.4 arcmin diameter area around the active region.

Our model calculations for the radio source show that two separate sources are needed to produce the observed flat spectra: one source with high-energy electrons and a second source with extremely high electron densities $\left(>10^{16} \mathrm{~m}^{-3}\right)$, large source dimensions $\left(>10^{15} \mathrm{~m}^{2}\right)$, and very low temperatures $\left(<10^{6} \mathrm{~K}\right)$. What this cool material consists of is unkown. Filaments are cool and dense but then they are also much more common than our type of $\mathrm{mm}$-wave rich events. The active role of cool $\left(10^{4} \mathrm{~K}\right)$ plasma during a flare, and without any observed presence of a prominence, has recently been reported by Kliem et al. (2002). We do not claim that our interpretation is the only imaginable one, but it is one possibility to explain these flat spectra in the context of direct emission processes. We propose multi-frequency and good spatial resolution radio imaging observations together with high-sensitivity observations in 
the $\mathrm{MeV}$ range to better determine what role exactly the cool, high-density plasma could play in the evolution of the gradual radio flares.

Acknowledgements. The authors wish to thank N. Vilmer, H. Hudson, and E. Valtaoja for helpful comments on the manuscript and the anonymous referee for improving the clarity of the paper. Yohkoh is a Japanese solar mission, with several internationally operated instruments. The archive of solar flare hard X-ray data from the BATSE experiment on the Compton Gamma Ray Observatory (CGRO) is part of the Solar Data Analysis Center (SDAC) at Goddard Space Flight Center. We thank the radio group at DASOP, Observatoire de Paris, France for the use of Nancay Radioheliograph data, and Metsähovi Radio Observatory, Helsinki Univ. of Technology, Finland, for the use of their radio data. S.P. was supported in Meudon by the Academy of Finland Contract No. 42576. J.H. was supported by DFG grant No. STA 351/6.

\section{References}

Aschwanden, M. J., Bynum, R. M., Kosugi, T., Hudson, H., \& Schwartz, R. A. 1997, ApJ, 487, 936
Benka, S. G., \& Holman, G. D. 1992, ApJ, 381, 854

Bornmann, P. L. 1990, ApJ, 356, 733

Chertok, I. M., Fomichev, V. V., Gorgutsa, R. V., et al. 1995, Sol. Phys., 160, 181

Dulk, G. A. 1985, ARA\&A, 23, 169

Emslie, A. G. 1978, ApJ, 224, 241

Hildebrandt, J., Krüger, A., Chertok, I. M., Fomichev, V. V., \& Gorgutsa, R. V. 1998, Sol. Phys., 181, 337

Hudson, H., \& Ryan J. 1995, ARA\&A, 33, 239

Kliem, B., Dammasch, I. E., Curdt, W., \& Wilhelm, K. 2002, ApJ, 568,61

Kundu, M. R., White, M., Gopalswamy, N., \& Lim, J. 1994, ApJS, 90, 599

Pohjolainen, S., \& Urpo, S. 1997, Proc. of the 5th SOHO Workshop, ESA-SP 404, 619

Pohjolainen, S., Valtaoja, E., \& Urpo, S. 1996, A\&A, 314, 947

Pohjolainen, S., Valtaoja, E., \& Urpo S. 1997, Adv. Space Res., 20, 12,2337

Ramaty, R. 1969, ApJ, 158, 753

Raulin, J.-P., White, S. M., Kundu, M. R., Silva, A. V. R., \& Shibasaki, K. 1999, ApJ, 522, 547

Thomas, R. J., Starr, R., \& Crannell, C. J. 1985, Sol. Phys., 95, 323

Urpo, S., Krüger, A., \& Hildebrandt, J. 1986, A\&A, 163, 340 